\title{
The Trapezius Muscle Flap: A Viable Alternative for Posterior Scalp and Neck Reconstruction
}

\author{
Hee Jun Yang ${ }^{1}$, Dong Hun Lee ${ }^{2}$, Yang Woo Kim², Sang Gu Lee ${ }^{3}$, Young Woo Cheon ${ }^{2}$ \\ Departments of ${ }^{1}$ Anatomy, ${ }^{2}$ Plastic and Reconstructive Surgery, and ${ }^{3}$ Neurosurgery, Gachon University Gil Medical Center, Gachon University \\ School of Medicine, Incheon, Korea
}

Background The trapezius muscle flap is not usually the first reconstructive option for skin and soft tissue defects in the posterior neck and scalp due to surgeons' unfamiliarity with the surgical anatomy and developments in free tissue transfer techniques. The goals of this study were to describe the clinical use of trapezius flaps in posterior neck and scalp reconstruction, and to investigate the vascular anatomy of trapezius flaps in Asians in order to obtain information facilitating the safe design and elevation of flaps in which most of the muscle is preserved.

Methods A retrospective chart review was performed of 10 patients who underwent trapezius muscle flap for posterior neck and scalp defects. We also performed an anatomical study of 16 flaps harvested from 8 preserved Asian adult cadavers and evaluated the main landmarks relevant for trapezius muscle flap.

Results In the anatomical study, the mean vertical height from the inferior angle of the scapula to the point at which the superficial cervical artery penetrated the trapezius was $4.31 \pm 2.14 \mathrm{~cm}$. The mean vertical height of the trapezius muscle flap pivot point was $9.53 \pm 2.08 \mathrm{~cm}$ from the external occipital protuberance. Among the 10 flaps, partial necrosis on the overlaid skin graft occurred in 1 patient and postoperative seroma occurred in another patient.

Conclusions Vascular variations in the trapezius muscle flap are uncommon in Asians, but when present, such variations appear to have little impact on harvesting the flap or on its circulation. The trapezius muscle flap is a viable alternative for posterior neck and scalp reconstruction.

Keywords Superficial back muscles / Surgical flap / Cadaver
Correspondence: Young Woo Cheon Department of Plastic and Reconstructive Surgery, Gachon University Gil Medical Center, Gachon University School of Medicine, 21 Namdong-daero 774beon-gil, Namdong-gu, Incheon 21565, Korea Tel: +82-32-460-2770

Fax: +82-32-460-3000

E-mail:youngwooc@gmail.com

The authors are grateful to Mrs. Hyun Joo Kim, BFA, for her help with the figures.

This research was supported by the Basic Science Research Program through the National Research Foundation of Korea (NRF), funded by the Ministry of Science, ICT \& Future Planning (NRF-

2014R1A1A1037258); a researchfocused grant from Gil Hospital (FRD 2013-41); and a research foundation grant from Gachon University (GCU2013-M055).

No potential conflict of interest relevant to this article was reported.

Received: 9 Jun 2016 • Revised: 22 Aug $2016 \bullet$ Accepted: 31 Aug 2016

pISSN: 2234-6163 • elSSN: 2234-6171 • https://doi.org/10.5999/aps.2016.43.6.529 • Arch Plast Surg 2016;43:529-535

\section{INTRODUCTION}

The trapezius flap was first reported by Nakajima and Fujino [1] in 1984. It was originally described as a myocutaneous or muscle flap, and it has also been used as a skin flap and free flap $[2,3]$. The trapezius myocutaneous flap is used in various salvage pro- cedures for head and neck reconstruction [4], in cases where previous radiation therapy and repeated surgical procedures limit the use of free flaps. However, the trapezius flap is not commonly used for reconstruction of the posterior neck and occipital scalp, as the relatively abundant neck and temporal vessels encourage the use of free flaps. Nevertheless, free flaps are less ap- 
propriate in frail older patients due to the longer operation time. In these patients, the trapezius flap could be a suitable alternative, as it is associated with a shorter operation time and minimal functional deficits. Furthermore, the trapezius muscle flap can provide a robust vascular supply, which may reduce the risk of infection in the presence of a prosthesis.

The trapezius muscle flap is also underused due to unfamiliarity with the surgical anatomy of the flap. According to the Mathes and Nahai classification, the trapezius muscle has a type II vascular pattern, with dominant and minor vascular pedicles [5]. The dominant vascular pedicle of the flap is derived from the transverse cervical artery (TCA) [6]. A descending branch of the TCA has been reported as a major vessel that ascends to the levator scapulae and rhomboid muscle to reach the trapezius [7]. In 1991, Netterville and Wood [8] reported that the dorsal scapular artery (DSA) was the dominant pedicle of the trapezius muscle based on their dissection of 15 cadavers. However, ongoing debate remains regarding whether the dominant vessel is a deep branch of the TCA [9]. Such debates may be due to frequent changes in anatomical nomenclature. Furthermore, most anatomical studies have been performed in Caucasians; therefore, the anatomy of the trapezius flap in Asians remains poorly understood.

The goal of the current study was to describe the clinical use of the trapezius flap for reconstruction of the posterior neck and scalp. We also investigated the vascular anatomy of the trapezius flap in Asians in order to obtain information facilitating the safe design and elevation of flaps in which most of the muscle is preserved.

\section{METHODS}

\section{Cadaver study}

In our anatomical study, we harvested 16 flaps from 8 preserved, Asian, adult cadavers and determined the following parameters: the vertical height of the trapezius muscle from its origin at the external occipital protuberance to its lowest point of origin, the vertical height of the trapezius muscle from its origin at the external occipital protuberance to the inferior angle of the scapula, the transverse distance from the midline to the point at which the superior cervical artery (SCA) penetrates the trapezius muscle, the level of SCA penetration into the trapezius muscle, and the length of the intramuscular portion of the SCA (viewed during dissection under loupes at $\times 2.5$ magnification). Finally, we evaluated the levels of the superficial and deep branches of the TCA in relation to the suggested pivot points for the trapezius muscle flap. All procedures were performed with the cadavers in the prone position. In order to allow exploration of the course of the SCA beneath the trapezius muscle, the intercostal vessels to the trapezius muscle were divided during muscle elevation.

\section{Patient characteristics}

A retrospective chart review was performed to identify patients who underwent trapezius muscle flap insertion for reconstruction of the posterior neck and occipital scalp area between January 2011 and August 2015. Ten consecutive patients underwent this procedure. The following data were obtained from their medical records: age, sex, cause and location of the defect, presence of previous irradiation at the site, pedicle vessel used, flap size, operation time, duration of follow-up, postoperative clinical course, and presence of complications.

\section{Operative technique}

Under general anesthesia, the patient was placed in the prone position on the operating table, and the expected margin of debridement or excision was marked. After adequate excision or debridement, the size of the defect was measured. The borders of the trapezius muscle, scapula, and scapular angle were marked for reference. A skin incision was created in a zigzag fashion to avoid scar contracture. A subcutaneous flap was elevated above the muscular fascia level to expose the medial and lateral border of the trapezius muscle. The lower portion of the muscle was detached along its lateral border and along the fibrous aponeurotic origin at the midline. Care was taken to preserve the underlying latissimus dorsi muscle during the elevation of the lowermost part of the trapezius. Submuscular dissection was performed in a caudal-to-cephalad direction. During this dissection, the most lateral attachment of the trapezius to the scapular spine and acromion was preserved. After submuscular dissection, the DSA, SCA, and accessory nerve were exposed.

The pedicle was selected according to the location and size of the defect. We preferred using SCA-pedicled trapezius flaps over DSA-pedicled flaps due to the wider rotation arc of the SCA-pedicled flaps, the fact that the long pedicle reaches as far as the orbital region of the face, and low donor site morbidity $[10,11]$. However, as the DSA is considered to have better blood flow during the operation than the SCA, if the DSA was found to result in an adequate pedicle length, we changed our original surgical plan and used a DSA-pedicled trapezius flap to cover the defect. The pivot point of the trapezius flap based on the SCA was at the level of the thyrocervical trunk in the lower neck. Since the TCA arises from the subclavian artery at the level of $\mathrm{T} 1$ and runs superior to the omohyoid muscle toward the anterior margin of the trapezius, the pivot point level was located between $\mathrm{C} 7$ and $\mathrm{T} 1$. If a longer pedicle was required, deep dissec- 
tion was performed through the SCA to the TCA. However, in our experience, deep dissection was not usually required (Fig. 1). The pivot point of flaps based on the DSA could vary in 2 ways. In some cases, the pivot point was located inferior to the rhomboid muscle, but this could limit the mobility of the flap. If a longer pedicle was required in such cases, division of the rhom- boid minor muscle was helpful $[11,12]$. Since the DSA is located more laterally than the SCA, the arc of rotation can be limited in DSA-based flaps. In other cases, the pivot point of DSAbased flaps was located between the rhomboid major and minor muscles (Fig. 2).

After selection of the appropriate pedicle, the muscle was divid-

Fig. 1. Posterior scalp defect (case 1, patient 9)

A 62-year-old woman presented with a skin and soft tissue defect involving an exposed implanted cranioplasty prosthesis (Medpor) on her posterior scalp. (A) The defect size was $4 \times 4 \mathrm{~cm}^{2}$. The exposed cranioplasty prosthesis was visible through the wound opening. (B) Following massive debridement due to a surrounding infection, a $27 \times 6-\mathrm{cm}^{2}$ trapezius muscle flap based on the superior cervical artery was elevated and transferred to the defect without subcutaneous tunneling. (C) The flap donor site and posterior neck incision for flap placement were closed with minimal subcutaneous undermining. An autologous split-thickness skin graft was laid on the remnant skin defect above the exposed muscle flap. (D) Seventeen-month postoperative results showing not only healing of the trapezius muscle flap and overlaid graft but also contouring with adequate thickness and good harmony with the surrounding tissue.
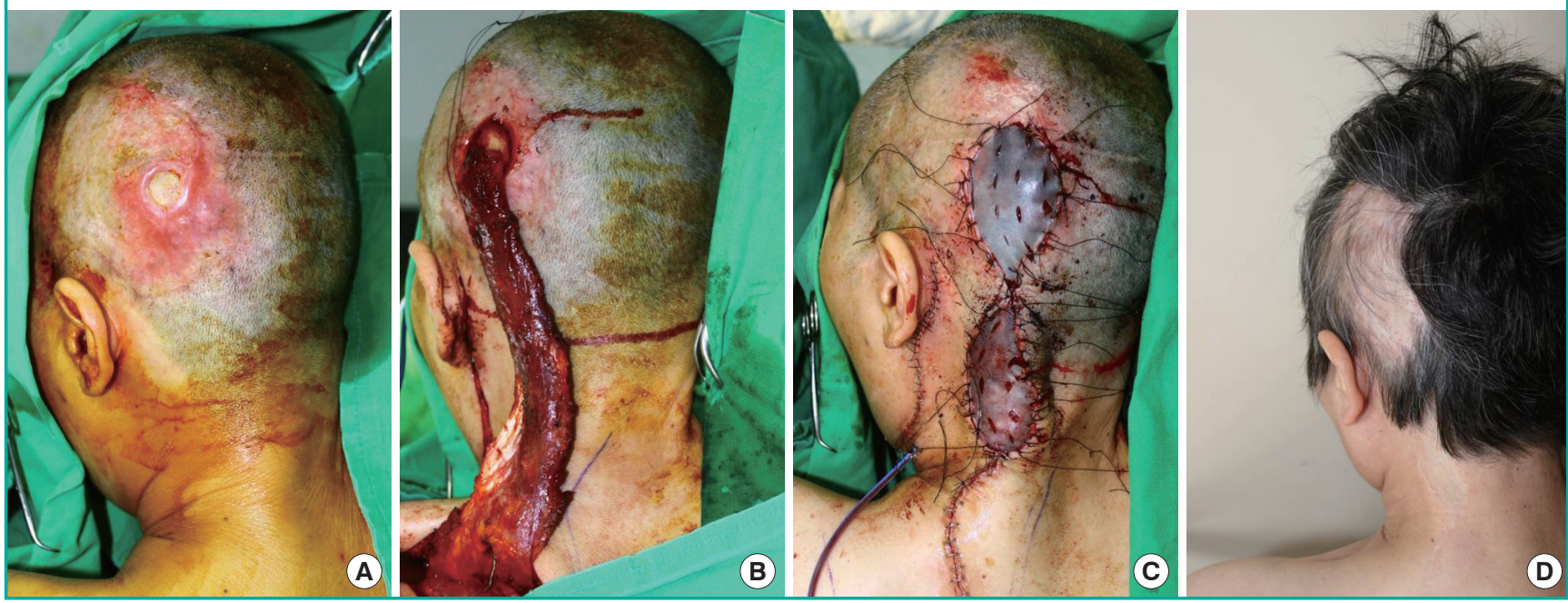

Fig. 2. Posterior neck defect (case 2, patient 10)

A 53-year-old man presented with a skin and soft tissue defect after cervical spine oncologic surgery. (A) The main problems were an exposed cervical spine metal fixation plate, deficiency of the covering soft tissue, and thin skin due to previous irradiation and repeated spinal surgery. (B) After spinal fusion surgery and excision of the devitalized tissue, an $18 \times 9-\mathrm{cm}^{2}$ trapezius muscle flap based on the dorsal scapular artery was elevated and rotated $160^{\circ}$ to cover the defect. The yellow arrow indicates the dorsal scapular artery-based pivot point. (C) The donor site and cephalic extended incision were closed without tension, and the remnant skin defect above the rotated trapezius muscle flap was covered with a split-thickness skin graft. (D) The trapezius flap and graft took well and showed adequate contouring.
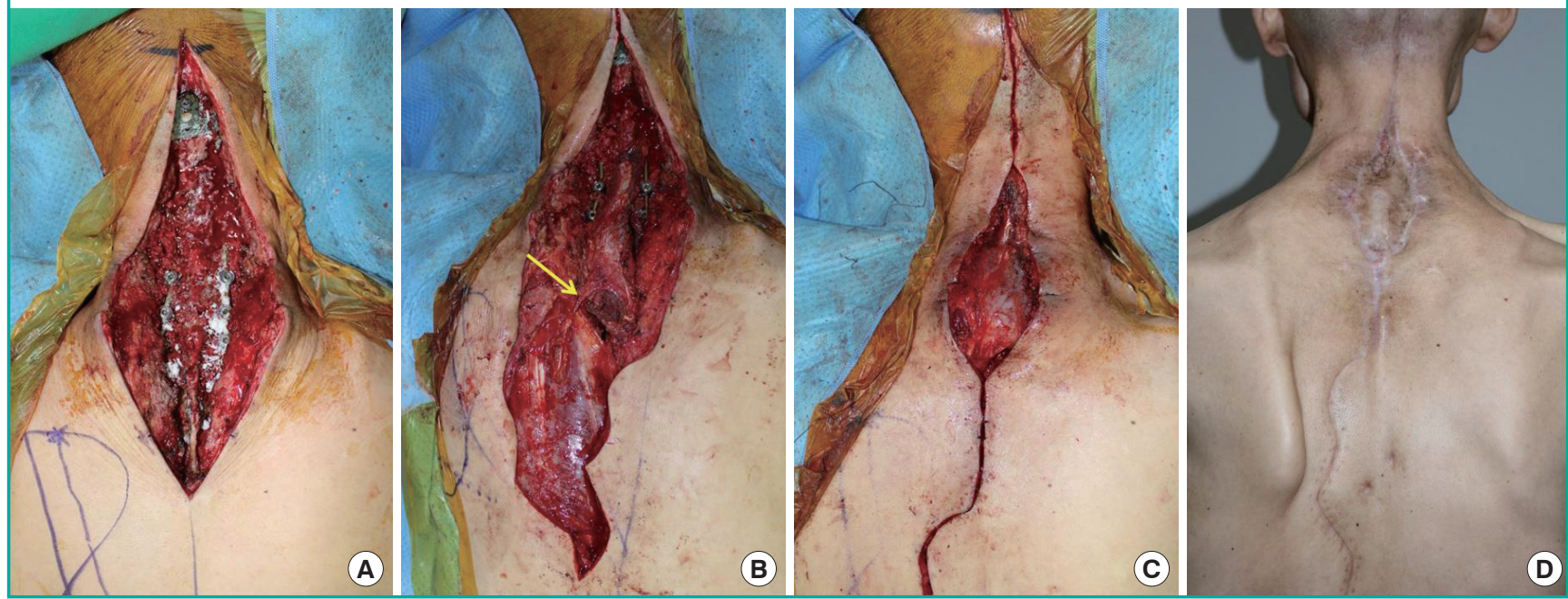
ed to reach the defect site. The superior part of the trapezius muscle was preserved to prevent shoulder drooping, pain, and weakness. The flap was then placed onto the defect, and the donor site was closed primarily with minimal subcutaneous undermining.

\section{Fig. 3. Anatomical parameters associated with trapezius} flap elevation

The trapezius muscle is detached from its medial origin to expose the superficial branch of the descending transverse cervical artery. (A) The mean vertical height of the medial side of the trapezius muscle origin was $35.85 \pm 1.86 \mathrm{~cm}$. (B) The mean vertical height from the uppermost aspect of the trapezius muscle origin to the inferior angle of the scapula was $24.59 \pm 1.47 \mathrm{~cm}$. (C) The mean transverse distance from the midline to the point at which the superior cervical artery (SCA) penetrated the trapezius muscle was $9.2 \pm 1.50 \mathrm{~cm}$. (D) Using loupe visualization, the length of the intramuscular portion of the SCA was noted to be $3.75 \pm 1.11 \mathrm{~cm}$. (E) The mean vertical height from the external occipital protuberance to the site of the SCA branch entering into the trapezius muscle was $20.28 \pm 2.47 \mathrm{~cm}$. (F) The mean vertical height from the external occipital protuberance to the trapezius muscle flap pivot point level was $9.53 \pm 2.08 \mathrm{~cm}$.

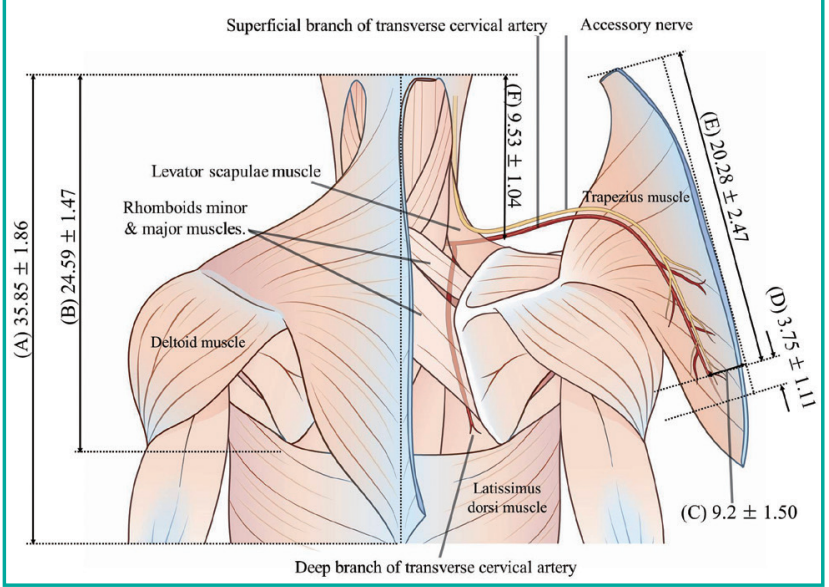

\section{RESULTS}

\section{Cadaver study}

We used 16 muscles from 8 preserved, adult, Asian cadavers to study the distribution of the SCA penetrating the trapezius muscle (Figs. 3,4$)$. The mean ( \pm standard deviation) vertical height of the medial side of the trapezius muscle origin was $35.85 \pm 1.86$ $\mathrm{cm}$, and the mean vertical height from the uppermost aspect of the trapezius muscle origin to the inferior angle of the scapula was $24.59 \pm 1.47 \mathrm{~cm}$. The average transverse distance from the midline to the point where the SCA penetrated the trapezius muscle was $9.2 \pm 1.50 \mathrm{~cm}$. The mean vertical height from the in-

Fig. 4. Cadaver dissection showing an elevated trapezius muscle

Yellow arrow, surgically divided rhomboid minor and levator scapulae muscles. Blue arrow, superficial cervical artery, showing its course beneath the anterior border of the trapezius muscle accompanied by the spinal accessory nerve.

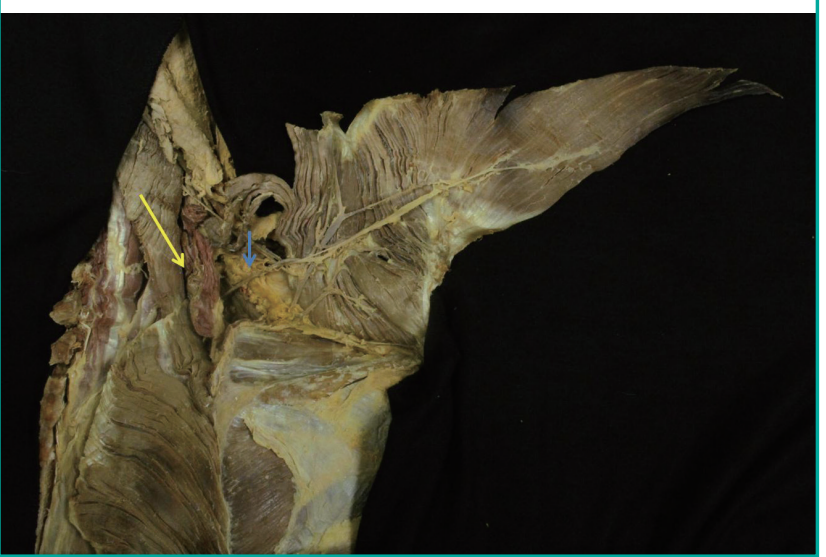

Table 1. Summary of cases

\begin{tabular}{|c|c|c|c|c|c|c|c|c|}
\hline $\begin{array}{l}\text { Case } \\
\text { no. }\end{array}$ & $\begin{array}{l}\text { Age }(y r) / \\
\text { Sex }\end{array}$ & Flap indication & $\begin{array}{l}\text { Defect } \\
\text { location }\end{array}$ & Pedicle & $\begin{array}{c}\text { Flap size, } \\
\text { (length } \times \text { width, } \\
\mathrm{cm})\end{array}$ & $\begin{array}{l}\text { Previous } \\
\text { irradiation }\end{array}$ & $\begin{array}{c}\text { Follow-up } \\
\text { (duration, mo) }\end{array}$ & Complications \\
\hline 1 & 49/Female & $\begin{array}{l}\text { Cervical spine prosthesis } \\
\text { infection }\end{array}$ & Posterior neck & SCA & $20 \times 8$ & Yes & 18 & None \\
\hline 2 & 76/Male & Metastatic SCC & Posterior neck & SCA & $19 \times 8$ & Yes & 16 & Tumor recurrence \\
\hline 3 & 67/Male & $\begin{array}{l}\text { Cranioplasty prosthesis } \\
\text { exposure }\end{array}$ & Occipital scalp & SCA & $26 \times 7$ & No & 22 & None \\
\hline 4 & 66/Female & Metastatic SCC & Posterior neck & DSA & $17 \times 7$ & Yes & 28 & None \\
\hline 5 & 52/Male & Sarcoma & Posterior neck & SCA & $20 \times 8$ & Yes & 23 & None \\
\hline 6 & 56/Male & Pressure sore & Occipital scalp & SCA & $24 \times 7$ & No & 21 & None \\
\hline 7 & 69/Male & Osteoradionecrosis & Occipital scalp & SCA & $28 \times 8$ & Yes & 20 & Donor site seroma \\
\hline 8 & 72/Male & Sarcoma & Posterior neck & DSA & $19 \times 8$ & Yes & 19 & Tumor recurrence \\
\hline 9 & 62/Female & $\begin{array}{l}\text { Cranioplasty prosthesis } \\
\text { exposure }\end{array}$ & Occipital scalp & SCA & $27 \times 6$ & No & 21 & None \\
\hline 10 & 53/Male & $\begin{array}{l}\text { Cervical spine prosthesis } \\
\text { infection }\end{array}$ & Posterior neck & DSA & $18 \times 9$ & Yes & 6 & None \\
\hline Mean & 62.2 & - & - & - & $21.8 \times 7.6$ & - & 19.4 & - \\
\hline
\end{tabular}


ferior angle of the scapula to the point where the SCA penetrated the trapezius muscle was $4.31 \pm 2.14 \mathrm{~cm}$. Using loupe visualization, the intramuscular portion of the SCA was noted to be $3.75 \pm 1.11 \mathrm{~cm}$. The mean vertical height from the external occipital protuberance to the trapezius muscle flap pivot point level was $9.53 \pm 2.08 \mathrm{~cm}$. The DSA (deep branch of the TCA) did not arise directly from the subclavian artery in any cadavers.

\section{Clinical results}

Of the 10 patients, 7 were male and 3 were female (Table 1 ). Their mean age was 62.2 years (range, $49-76$ years). The causes of the skin and soft tissue defects in these patients are shown in Table 1 . Seven patients had previously undergone radiation therapy for an underlying disease. Of the 10 flaps, 7 were SCA-pedicled flaps and 3 were DSA-pedicled flaps. In 2 patients, recurrence of the underlying disease was found in the surgical specimens. The mean postoperative follow-up period was 19.4 months (range, 6-28 months). The flaps had a mean width of $7.6 \mathrm{~cm}$, a mean length of $21.8 \mathrm{~cm}$, and a mean area of $164.1 \mathrm{~cm}^{2}$ (range, $119-224 \mathrm{~cm}^{2}$ ) (Table 1). Eight flaps survived without complications. One patient (case 2 below) developed a minor complication requiring revisional surgery. Another patient developed a postoperative seroma that healed with conservative treatment.

\section{DISCUSSION}

The anatomy of the trapezius muscle has been extensively described [13]. The trapezius muscle is one of the largest muscles in the body. It is divided into 3 parts according to function and the direction of the muscle fibers: superior (descending), middle (transverse), and inferior (ascending). The superior part originates from the spinous process of the seventh cervical vertebra (C7), the external occipital protuberance, and the ligamentum nuchae. From this origin, fibers proceed downward and laterally and are inserted into the posterior border of the lateral third of the clavicle. The middle part arises from the spinous processes of $\mathrm{C} 7$ and the first three thoracic vertebrae (T1-3) and inserts into the medial margin of the acromion and the superior lip of the posterior border of the spine of the scapula. The inferior part arises from the spinous processes of the remaining thoracic vertebrae and proceeds upward and laterally to converge near the scapula and end in an aponeurosis. The trapezius muscle has 2 main functions: movement of the scapula when the spinal segments are stable, and movement of the spine when the scapula is stable. It is innervated by the spinal accessory nerve (the 11th cranial nerve). Disruption of the spinal accessory nerve, which supplies trapezius motor innervation, produces shoulder drooping or limited arm rotation. The dominant blood supply of the trapezius muscle and overlying skin, considering its variations, is provided by the superficial (SCA) and deep (DSA) branches of the TCA; the TCA generally arises from the thyrocervical trunk [14]. The proportion of the dominance of these 2 vessels in the muscle has been reported to vary across studies $[8,11]$. The superficial branch is also known as the superficial cervical artery (SCA) [2]; this nomenclature has been constant over time, and therefore has not led to any significant misunderstandings. In contrast, confusion has occurred due to changes in the nomenclature of the deep branch of the TCA. Most recent articles have described the deep branch as the DSA [14]. However, some articles describe the DSA as an independent vessel arising directly from the subclavian artery [15], which can be observed as an uncommon anatomical variation [11].

The anatomy of the TCA in the posterior triangle is highly variable $[12,14]$. The TCA enters the trapezius muscle at the base of the neck and descends vertically along the deep surface of the muscle. It arises directly from the subclavian artery in most people and extends across the posterior triangle of the neck where it divides into the SCA, which crosses over the levator scapulae, and a deep branch (the DSA), which runs deep to the levator scapulae [14]. The SCA gives rise to a descending branch and an ascending branch, and the DSA gives rise to a superficial branch that penetrates between the rhomboid minor and major muscles and a deep branch that descends under the rhomboid major muscle. The SCA gives rise to ascending and descending branches, which can be used individually when raising a trapezius muscle flap. Minor pedicles arising from the occipital artery, circumflex scapular artery, and intercostal perforators are uncommonly used for trapezius flaps.

The SCA runs lateral and superficial to the levator scapulae and rhomboid muscles and is accompanied by branches of the accessory nerve [10]. Its course lies beneath the anterior border of the trapezius muscle. The trapezius muscle flap is designed along the course of the SCA. Thus, the long axis of the flap is centered between the spine and the medial border of the scapula. The medial border of the flap is marked $1.5 \mathrm{~cm}$ lateral to the spinous processes, and the width of the flap is generally $6-9 \mathrm{~cm}$. The pivot point of the flap is positioned at the level of the scapular spine to preserve the superior portion of the trapezius muscle. Our cadaver study demonstrated that the pivot point was usually at the level of the $\mathrm{C} 7$ spinous process, at a vertical height from the external occipital protuberance of $9.53 \pm 2.08 \mathrm{~cm}$. Additionally, the SCA branch to the trapezius muscle was found to be positioned at the level of the inferior angle of the scapula. These anatomical findings can help surgeons to design the flap and to perform the surgery more quickly. The distance between the pivot point and defect determines the pedicle length. The 
length of the SCA anterior to and within the trapezius muscle in our cadaver study suggested that the minimum safe pedicle length is $14.5 \pm 1.83 \mathrm{~cm}$ from the pivot point. Clinically, the flap pedicle length used in our patients was longer than the mean length suggested by the results of the cadaver study, and no circulation-related complications occurred.

The trapezius flap is versatile, practical, and reliable [16]. It is multipurpose, and according to the wound status, it can range from a muscle-only flap to a perforator-based skin flap, and can even be used as a bilateral flap or an osteomusculocutaneous trapezius flap [17-19]. The various methods used in trapezius muscle flaps contribute to its usefulness as an option for head and neck reconstruction. The authors of the present study preferred trapezius muscle flaps covered with an overlaid skin graft instead of trapezius flaps including a skin paddle, such as myocutaneous or fasciocutaneous flaps, since the muscle flap has very robust circulation with adequate thickness, allowing it to be more resistant to infections than other cutaneous flaps. Additionally, myocutaneous flaps are transferred with more volumefilling components than muscle-only flaps with overlaid skin grafts. Although the muscle portion of the flap should atrophy later, the bulkiness of the flap could cause patients to experience discomfort when they move their neck. However, no patients in our study suffered from contracture or limitations of neck motion.

Trapezius muscle flaps exhibit the advantages of local flaps in comparison to distant tissue transfer, including an easier surgical technique; better matching with the surrounding tissue in terms of skin color, texture, and thickness; and a shorter operation time. Although various free tissue transfer methods have been described for reconstruction $[20,21]$, microvascular surgery is not a good option in a previously irradiated surgical field. Local flaps are not contraindicated in such situations. Trapezius muscle flaps also have the advantage of allowing all procedures (flap elevation, recipient site preparation, and flap placement) to occur with the patient in the prone position. This makes the operation easier and shorter. Furthermore, the trapezius flap can rotate in a wide arc to reach virtually any defect in the occiput, nuchal, or spinal areas, and it is thinner and more pliable than both latissimus dorsi and pectoralis major flaps [4]. The donor site can usually be closed directly, and donor site-associated morbidity is minimal. In addition, the donor site scar is in the posterior trunk, which is easily concealed under clothes. Functional impairment does not occur if the upper part of the trapezius muscle and rhomboid muscles containing the spinal accessory nerve are preserved. Furthermore, the trapezius flap can act as a back-up option if pedicle damage occurs during harvesting of a thoracodorsal artery perforator flap, as the trapezius perforators are located within the same surgical field as those of the thoracodorsal artery [12].
The main drawback of the trapezius muscle flap is its limited width, which is restricted to a maximum of $8-10 \mathrm{~cm}$. The width is determined by pinching the donor site skin to be closed primarily. If a larger flap with more muscle mass or a longer length (distance from the pivot point to the defect) is required, the risk of donor site complications can be quite high and marginal flap circulation may be compromised [11]. When the trapezius muscle flap is based on the DSA, division of the rhomboid minor muscle can lengthen the pedicle $[11,12]$. Usually SCA-pedicled flaps do not require additional length; however, if a longer pedicle is required, dissection can be performed above the rhomboid to the scalene muscle, with division of the DSA. When elevating the trapezius muscle from the midline, the approximately $1.5 \mathrm{~cm}$-wide aponeurosis attachment to the spinous processes is left intact, as the flap does not require this thin fibrous tissue.

A postoperative donor site seroma occurred in 1 of our 10 patients. No functional deficits of the trapezius were observed, which we attribute to preservation of the upper part of the trapezius muscle [9]. No flap necrosis was observed. The flaps were approximately $11 \mathrm{~cm}$ longer than the average distance at which the SCA penetrated the trapezius muscle, but no flap circulation problems occurred. In the clinical setting, the part of the flap that extended beyond this end point of SCA within the trapezius muscle (measuring, on average, approximately $7 \mathrm{~cm}$ ) appears to be safe.

A limitation of this study is that we did not assess the vascular anatomy of the DSA by measuring parameters such as its length, vertical level, and surface landmarks, although the DSA is one of the major pedicles of the trapezius muscle flap. Further study is needed to evaluate the vascular anatomy of the DSA.

Trapezius muscle flaps are not widely used due to vascular variations and confusion regarding the anatomical nomenclature. However, vascular variations appear to be uncommon in Asians, and if such variations are present, they have little impact on harvesting the flap or on its circulation. The trapezius muscle flap is, thus, a viable alternative for posterior neck and scalp reconstruction.

\section{ORCID}

Dong Hun Lee http://orcid.org/0000-0001-9870-8266

\section{REFERENCES}

1. Nakajima $\mathrm{H}$, Fujino T. Island fasciocutaneous flaps of dorsal trunk and their application to myocutaneous flap. Keio J Med 1984;33:59-82.

2. Hyakusoku H, Yoshida H, Okubo M, et al. Superficial cervi- 
cal artery skin flaps. Plast Reconstr Surg 1990;86:33-8.

3. Hyakusoku H, Takizawa Y, Murakami M, et al. Versatility of the free or pedicled superficial cervical artery skin flaps in head and neck burns. Burns 1993;19:168-73.

4. Ugurlu K, Ozcelik D, Huthut I, et al. Extended vertical trapezius myocutaneous flap in head and neck reconstruction as a salvage procedure. Plast Reconstr Surg 2004;114:33950.

5. Mathes SJ, Nahai F. Classification of the vascular anatomy of muscles: experimental and clinical correlation. Plast Reconstr Surg 1981;67:177-87.

6. Rosen HM. The extended trapezius musculocutaneous flap for cranio-orbital facial reconstruction. Plast Reconstr Surg 1985;75:318-27.

7. Chandrasekhar B, Terz JJ, Kokal WA, et al. The inferior trapezius musculocutaneous flap in head and neck reconstruction. Ann Plast Surg 1988;21:201-9.

8. Netterville JL, Wood DE. The lower trapezius flap: vascular anatomy and surgical technique. Arch Otolaryngol Head Neck Surg 1991;117:73-6.

9. Lynch JR, Hansen JE, Chaffoo R, et al. The lower trapezius musculocutaneous flap revisited: versatile coverage for complicated wounds to the posterior cervical and occipital regions based on the deep branch of the transverse cervical artery. Plast Reconstr Surg 2002;109:444-50.

10. Ogawa R, Murakami M, Vinh VQ, et al. Clinical and anatomical study of superficial cervical artery flaps: retrospective study of reconstructions with 41 flaps and the feasibility of harvesting them as perforator flaps. Plast Reconstr Surg 2006;118:95-101.

11. Haas F, Weiglein A, Schwarzl F, et al. The lower trapezius musculocutaneous flap from pedicled to free flap: anatomical basis and clinical applications based on the dorsal scapu- lar artery. Plast Reconstr Surg 2004;113:1580-90.

12. Sadigh PL, Chang LR, Hsieh CH, et al. The trapezius perforator flap: an underused but versatile option in the reconstruction of local and distant soft-tissue defects. Plast Reconstr Surg 2014;134:449e-456e.

13. Yang D, Morris SF. Trapezius muscle: anatomic basis for flap design. Ann Plast Surg 1998;41:52-7.

14. Can A, Orgill DP, Dietmar Ulrich JO, et al. The myocutaneous trapezius flap revisited: a treatment algorithm for optimal surgical outcomes based on 43 flap reconstructions. J Plast Reconstr Aesthet Surg 2014;67:1669-79.

15. Angrigiani C, Grilli D, Karanas YL, et al. The dorsal scapular island flap: an alternative for head, neck, and chest reconstruction. Plast Reconstr Surg 2003;111:67-78.

16. Wang J, Zhao Z, Yu L, et al. The extra-long artery-pedicled back fascia skin flap for treatment of cervical contractive scar. J Plast Reconstr Aesthet Surg 2006;59:331-6.

17. Hafezi F, Naghibzadeh B, Pegahmehr M, et al. Extended vertical trapezius fasciocutaneous flap (back flap) in face and neck burn scar reconstruction. Ann Plast Surg 2008;61:441-6.

18. Vacher C, de Vasconcellos JJ. The anatomical basis of the osteo-musculo-cutaneous trapezius flap in mandibular reconstruction. Surg Radiol Anat 2005;27:1-7.

19. Dufresne C, Cutting C, Valauri F, et al. Reconstruction of mandibular and floor of mouth defects using the trapezius osteomyocutaneous flap. Plast Reconstr Surg 1987;79:68796.

20. Lutz BS, Wei FC, Chen HC, et al. Reconstruction of scalp defects with free flaps in 30 cases. Br J Plast Surg 1998;51: 186-90.

21. Disa JJ, Pusic AL, Hidalgo DH, et al. Simplifying microvascular head and neck reconstruction: a rational approach to donor site selection. Ann Plast Surg 2001;47:385-9. 